\title{
The involvement of TREM2 R47H variant in Alzheimer disease confirmed, but mechanisms remain elusive
}

genetic study of over 173,000
individuals has confirmed
that the TREM2 amino-acidsubstitution mutation $\mathrm{R} 47 \mathrm{H}$ increases the risk of Alzheimer disease (AD) in people of European descent. The results, published in Alzheimers \& Dementia, suggest the mutation contributes through tau dysfunction.

Several studies have reported the TREM2 R47H mutation to be a risk factor for $\mathrm{AD}$, but the magnitude of this mutation's role in $\mathrm{AD}$ and other neurodegenerative diseases has been far from clear. "The effect size estimates varied widely across datasets," says lead author Christina Lill. "We aimed to derive a realistic estimate for $\mathrm{R} 47 \mathrm{H}$ in $\mathrm{AD}$ and to determine whether it is also associated with other neurodegenerative disorders." Lill and colleagues genotyped 19,940 participants of European ancestry, including patients with neurodegenerative disorders, as well as healthy control individuals. The investigators then combined these novel data with 28 previously published data sets, yielding a total European-origin population of 24,086 individuals with $\mathrm{AD}$ and 149,993 controls. In this population, TREM2 $\mathrm{R} 47 \mathrm{H}$ mutation was associated with a substantial increase $(\mathrm{OR}=2.71)$ in risk of $\mathrm{AD}$.

In contrast with some previous reports, Lill and co-workers found no association between the mutation and the risk of Parkinson disease (PD), amyotrophic lateral sclerosis (ALS), or frontotemporal lobar degeneration

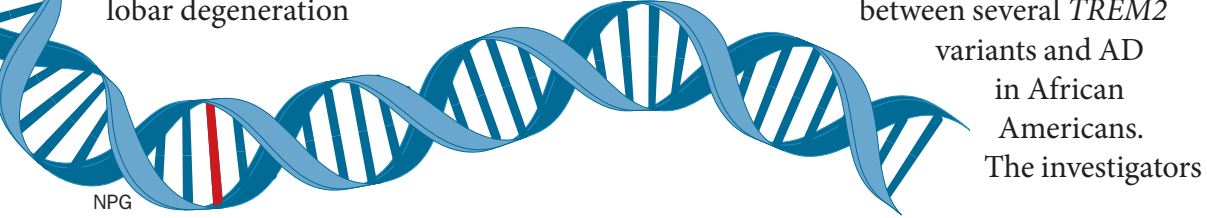

(FTLD). "Although our calculations and conclusions are based on the largest sample sizes analysed for each of the four diseases to date, it needs to be emphasized that the sample sizes for PD, FTLD, and ALS were substantially smaller than the data available for AD," notes Lill. "Thus, we cannot exclude the presence of much smaller effects for these diseases."

The investigators also assessed the levels of amyloid- $\beta_{42}\left(A \beta_{42}\right)$ and total tau in the cerebrospinal fluid (CSF) of 828 individuals with $\mathrm{AD}$ or mild cognitive impairment. The R47H mutation was linked to an increased CSF level of total tau, but not $A \beta_{42}$. "This finding is in line with a previously published study by another group, and suggests that the role of TREM2 in AD pathophysiology predominantly involves tau dysfunction," says Lill.

"The genetic link between AD and TREM2 can now be considered established," contends Lars Bertram, who led the study. However, two other recent investigations suggest that the importance of TREM2 variants as risk factors for AD depends on the population.

The study by Lill et al. focused exclusively on datasets with people of European descent. In a study, published in American Journal of Alzheimer Disease and Other Dementias, Man Huang and co-investigators evaluated the association between TREM2 and AD in Japanese, Korean and Chinese people. Huang et al. conducted a meta-analysis of five studies comprising 3,962 individuals with $\mathrm{AD}$ and 4,403 healthy controls, and found no association between AD and the TREM2 $\mathrm{R} 47 \mathrm{H}$ mutation in East Asian populations. In another study, published in Molecular Neurodegeneration, Sheng Chih Jin and colleagues evaluated the links between several TREM2 ants and $\mathrm{AD}$ in African Americans The investigators reported that the $\mathrm{R} 47 \mathrm{H}$ mutation was not associated with a significant increase in risk of $\mathrm{AD}$, although the authors note that the study could have been underpowered to detect such a link. Instead, Jin et al. found that other TREM2 mutations were relevant to the risk of $\mathrm{AD}$ in the African American population. This finding is consistent with a previous study linking several TREM2 shortnucleotide polymorphisms with $\mathrm{AD}$ in people of African descent.

"Most papers published to date-including ours-have only focused on the $\mathrm{R} 47 \mathrm{H}$ variant in TREM2. There are, however, other nucleotide substitutions in this gene, some of which are predicted to have functional consequences," says Bertram. Future studies should assess whether these additional variants also show association with $\mathrm{AD}$ or other neurodegenerative diseases.

Moreover, little is known about the precise biochemical mechanisms that underlie the association between $\mathrm{AD}$ and TREM2. According to Bertram, studies on cellular and animal models are needed to shed new light on the pathophysiological processes through which TREM2 variants confer a risk for $\mathrm{AD}$.

\section{Hemi Malkki}

